\title{
Bilateral Mastectomy: Doubling Down on Complications?
}

\author{
Todd M. Tuttle, MD and Erin E. Burke, MD \\ Division of Surgical Oncology, University of Minnesota, Minneapolis, MN
}

Complications after breast cancer surgery can result in increased hospitalization, additional surgical and medical interventions, increased costs, and delays in initiating recommended adjuvant treatment. Moreover, complications may lead to long-term emotional distress and patient dissatisfaction. Thus, accurate information regarding the risk, severity, and timing of postoperative complications must be clearly presented to breast cancer patients. In the accompanying study by Silva and colleagues, the authors used the American College of Surgeons National Surgery Quality Improvement Program (NSQIP) to compare the rates of postoperative complications between unilateral and bilateral mastectomy among patients who underwent immediate reconstruction. ${ }^{1}$ The authors reported that bilateral mastectomy was associated with longer hospital stays and higher transfusion rates than unilateral mastectomy. Also, bilateral mastectomy with implant reconstruction was associated with a modestly increased reoperation rate. Surprisingly, surgical site infections, prosthesis failure, and medical complications occurred at similar rates between unilateral and bilateral mastectomy. For implant-reconstructed patients, the overall complication rate was $8.8 \%$ after unilateral mastectomy and $10.1 \%$ after bilateral mastectomy. The rates of surgical site complications were 4.2 and $4.6 \%$, respectively.

Determining the rates of postoperative complications after breast cancer surgery can be a frustrating endeavor. Published studies vary widely in regards to the definition, inclusion, and severity of postoperative complications. In addition, the length of follow-up from different studies ranges from a few weeks to a few years. As a result, the published rates of postoperative complications after breast

(C) Society of Surgical Oncology 2015

First Received: 30 April 2015;

Published Online: 21 May 2015

T. M. Tuttle, MD

e-mail: tutt1006@umn.edu surgery vary dramatically from study to study. Even the results from different studies using the same database seem incongruent. For example, Silva and colleagues report the 30-day surgical site complication rate after bilateral mastectomy and implant-based reconstruction as only $4.6 \%$. In a very similar study also using NSQIP, Osman and colleagues report the 30-day surgical site complication rate after bilateral mastectomy without reconstruction as $5.8 \%$. $^{2}$ Is the surgical site complication rate really lower for mastectomy after implant-based reconstruction as compared with mastectomy without reconstruction? Also, in the report by Osman and colleagues, the risk of postoperative complications was almost double after bilateral mastectomy as compared to unilateral mastectomy.

The 30-day outcomes reported by Silva and colleagues likely substantially underestimate the overall postoperative complication rates after bilateral mastectomy and reconstruction. In a multicenter study from the Cancer Research Network, Barton and colleagues determined the complication rates after bilateral mastectomy. ${ }^{3}$ In their patient cohort, $69.1 \%$ underwent implant reconstruction, and almost two-thirds of the women undergoing bilateral mastectomy had at least one complication. ${ }^{3}$ In a singlecenter study of 600 patients $(58 \%$ had implant reconstruction), Miller and colleagues reported that the overall complication rate after bilateral mastectomy was $41.6 \%$; on multivariate analysis, bilateral mastectomy was associated with a 2.7 times increased risk of major complications as compared to unilateral mastectomy. ${ }^{4}$ In another study that included 593 patients who underwent implant-based reconstruction after bilateral mastectomy, Zion and colleagues reported that $52 \%$ of patients required at least one unanticipated reoperation during a median follow-up of 14 years. ${ }^{5}$ Approximately $39 \%$ of all reoperations occurred within 1 year of breast reconstruction.

Silva and colleagues appropriately list a number of potential limitations related to NSQIP when it comes to analyzing complication rates after bilateral mastectomy. 
NSQIP likely underestimates the true complication rate because it does not include outpatient management of complications, is limited to 30-day follow-up, and it does not capture some complications that are specific to breast reconstruction. The limitations of NSQIP in capturing clinically relevant postoperative complications have also been noted for melanoma, thyroid, and pancreatic surgery. ${ }^{6-8}$ In another study of patients undergoing breast reconstruction or breast reduction, Khavanin and colleagues compared NSQIP to the Tracking Operations and Outcomes for Plastic Surgeons (TOPS) database. ${ }^{9}$ The authors found that compared to TOPS, NSQIP missed surgical complications and was not able to define the complications as specifically as TOPS.

Many studies, including the accompanying study by Silva and colleagues, compare the surgical complication rates between unilateral and bilateral mastectomy, with or without reconstruction. However, a large proportion of the patients who undergo bilateral mastectomy today actually have stage I/II breast cancer. For many of these patients, a more meaningful comparison is breast-conserving surgery versus bilateral mastectomy. Compared to bilateral mastectomy, breast-conserving surgery is clearly associated with a much shorter operating time, less hospitalization, fewer complications, less drains, and shorter overall recovery time. In addition, for the vast majority patients with unilateral breast cancer, bilateral mastectomy does not provide any meaningful survival benefit. Given the current limitations with NSQIP, physicians must be careful in interpreting the data from this study by Silva and colleagues when counseling women about their risks of complications after bilateral mastectomy.
DISCLOSURE The authors declare no conflict of interest.

\section{REFERENCES}

1. Silva A, Lapin B, Yao K, Song DH, Sisco M. The effect of contralateral prophylactic mastectomy on perioperative complications in women undergoing immediate breast reconstruction: a NSQIP analysis. Ann Surg Oncol (in press).

2. Osman F, Saleh F, Jackson TD, Corrigan MA, Cil T. Increased postoperative complications in bilateral mastectomy patients compared to unilateral mastectomy:an analysis of the NSQIP database. Ann Surg Oncol. 2013;20:3212-7.

3. Barton MB, West CN, Liu IL, et al. Complications following bilateral prophylactic mastectomy. J Natl Cancer Inst Monogr. 2005;(35):61-6.

4. Miller ME, Czechura T, Martz B, et al. Operative risks associated with contralateral prophylactic mastectomy: a single institution experience. Ann Surg Oncol. 2013;20:4113-20.

5. Zion SM, Slezak JM, Sellers TA, et al. Reoperations after prophylactic mastectomy with or without implant reconstruction. Cancer. 2003;98:2152-60.

6. Glarner CE, Greenblatt DY, Rettammel RJ, Neuman HB, Weber SM. Wound complications after inguinal lymph node dissection for melanoma: is ACS NSQIP adequate? Ann Surg Oncol. 2013;20:2049-55.

7. Sippel RS, Chen H. Limitations of the ACS NSQIP in thyroid surgery. Ann Surg Oncol. 2011;18:3529-30.

8. Epelboym I, Gawlas I, Lee JA, Schrope B, Chabot JA, Allendorf JD. Limitations of ACS-NSQIP in reporting complications for patients undergoing pancreatectomy: underscoring the need for a pancreas-specific module. World J Surg. 2014;38:1461-7.

9. Khavanin N, Gutowski KA, Hume KM, et al. The use of patient registries in breast surgey: a comparison of the Tracking Operations and Outcoms for Plastic Surgeons and National Surgical Quality Improvement Program data sets. Ann Plast Surg. 2015;74:157-62. 